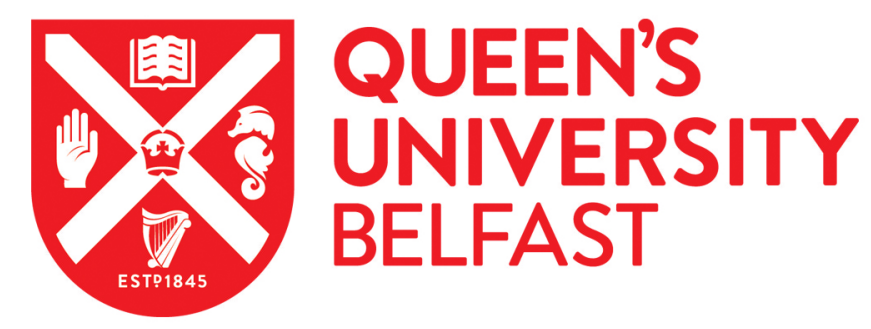

\title{
Electronically controlled surface plasmon dispersion and optical transmission through metallic hole arrays using liquid crystal.
}

Pollard, R., Dickson, W., Wurtz, G. A., Evans, P. R., \& Zayats, A. V. (2008). Electronically controlled surface plasmon dispersion and optical transmission through metallic hole arrays using liquid crystal. Nano Letters, 8(11)(1), 281-286. https://doi.org/10.1021/nl072613g

Published in:

Nano Letters

Queen's University Belfast - Research Portal:

Link to publication record in Queen's University Belfast Research Portal

\section{General rights}

Copyright for the publications made accessible via the Queen's University Belfast Research Portal is retained by the author(s) and / or other copyright owners and it is a condition of accessing these publications that users recognise and abide by the legal requirements associated with these rights.

Take down policy

The Research Portal is Queen's institutional repository that provides access to Queen's research output. Every effort has been made to ensure that content in the Research Portal does not infringe any person's rights, or applicable UK laws. If you discover content in the Research Portal that you believe breaches copyright or violates any law, please contact openaccess@qub.ac.uk. 


\title{
Electronically Controlled Surface Plasmon Dispersion and Optical Transmission through Metallic Hole Arrays Using Liquid Crystal
}

2008

Vol. 8, No. 1

281-286

\author{
Wayne Dickson, * Gregory A. Wurtz, Paul R. Evans, Robert J. Pollard, and \\ Anatoly V. Zayats \\ Centre for Nanostructured Media, IRCEP, The Queen's University of Belfast, \\ Belfast BT7 1NN, United Kingdom
}

Received October 10, 2007; Revised Manuscript Received November 19, 2007

\begin{abstract}
The enhanced optical properties of metal films periodically perforated with an array of sub-wavelength size holes have recently been widely studied in the field of surface plasmon optics. The ability to design the optical transmission of such nanostructures, which act as plasmonic crystals, by varying their geometrical parameters gives them great flexibility for numerous applications in photonics, opto-electronics, and sensing. Transforming these passive optical elements into devices that may be actively controlled has presented a new challenge. Here, we report on the realization of an electrically controlled nanostructured optical system based on the unique properties of surface plasmon polaritonic crystals in contact with a liquid crystal (LC) layer. We discuss the effect of LC layer modulation on the surface plasmon dispersion, the related optical transmission and the underlying mechanism. The reported effect may be used to achieve active spectral tuneability and switching in a wide range of applications.
\end{abstract}

Nanoplasmonics provides a pathway to both control and manipulate optical signals by coupling them to coherent electronic excitations near a metal surface. ${ }^{1}$ This has led to the development of a toolbox of various subwavelength photonic components such as mirrors, lenses, plasmonic crystals, and waveguides capable of manipulating plasmonic signals. ${ }^{1,2}$ Moreover, the plasmonic properties of metallic nanostructures determines their conventional optical properties such as reflection, transmission, and absorption. Plasmonic crystals ${ }^{3,4}$ are considered as the basis for superlenses, 5,6 metamaterials for negative refraction applications, ${ }^{7,8}$ as well as nonlinear metamaterials with enhanced optical nonlinearity. ${ }^{9,10}$ Plasmonic nanostructures are becoming important constituents in light-emitting devices (LEDs, OLEDs, nanolasers), photodetectors, and nanoscale light sources as well as the principle components for future optical data storage, imaging, and sensing.

One of the most important yet challenging requirements for applications in photonics, opto-electronics, and optical communications is to introduce a means to control the optical properties of plasmonic devices. To directly influence surface plasmons and hence the associated optical properties, manipulation of the refractive index of the dielectric medium adjacent to the metal surface is the method of choice. The

\footnotetext{
* Corresponding author. Email: w.dickson@qub.ac.uk.
}

route to all-optical control takes advantage of the intense nature of confined electromagnetic radiation in nanostructured elements as the stimulus for optically nonlinear dielectrics that are hybridized with the metallic structure..$^{10,11}$ Another technique of fundamental importance in the context of controlling surface plasmon polaritonic (SPP) signals is the application of an external electric field. ${ }^{12}$ This is especially significant because both plasmonic and electric signals can be guided in the same metallic circuitry. The applied voltage can then be used to exert direct control over an electro-optically active dielectric medium changing its refractive index and thus the SPP modes on the metal/ dielectric interface.

Liquid crystals (LCs) are an excellent example of such a dielectric and are well known through their use in display technology. The large broadband optical anisotropy of these molecules makes them ideal candidates for electrically operated nanoplasmonic devices. Indeed, experiments have already been carried out on the modification of the surface plasmon resonances of smooth silver films coated with liquid crystal, ${ }^{13}$ variation of the localized plasmonic resonance of gold nanoparticles embedded in liquid crystal host ${ }^{14}$ as well as ensembles of gold nanorods aligned both paralle ${ }^{15}$ and perpendicular $^{16}$ to a substrate. The application of liquid crystal molecules to the case of a periodically nanostructured 


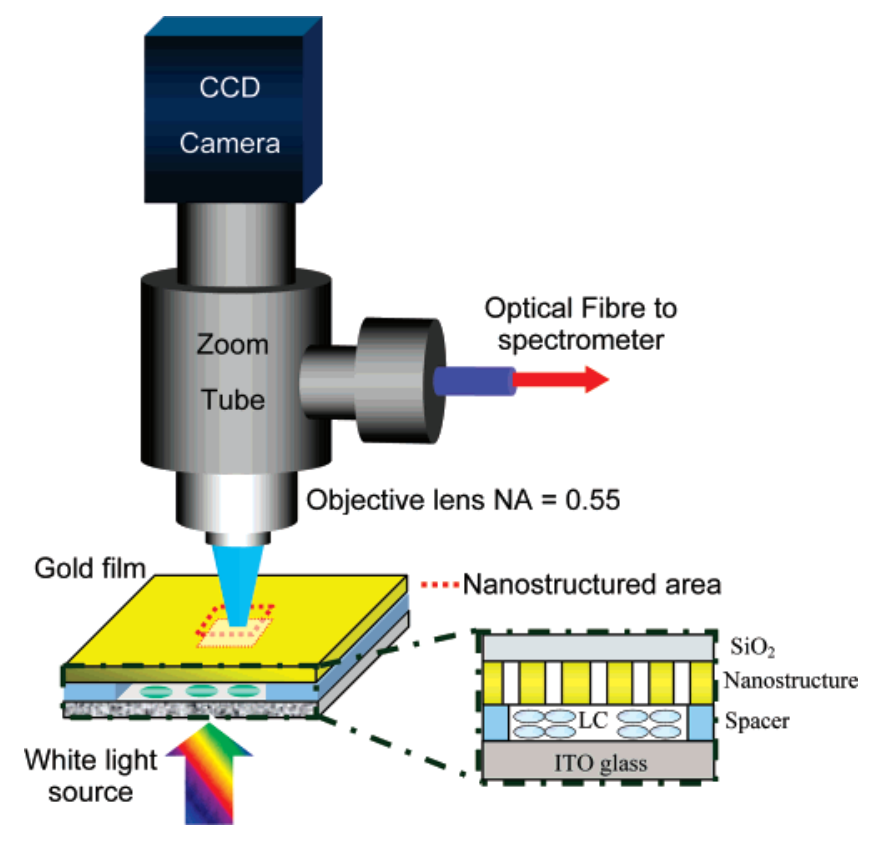

Figure 1. Schematic of experimental arrangement. Collimated white light is incident on the liquid crystal cell passing first through the ITO glass and liquid crystal before illuminating the structured area. Light is collected by the objective lens and split into two paths delivering light to both the CCD camera and spectrometer.

metallic film may therefore lead to the development of electronically controlled enhanced transmission, reflection, and absorption of the plasmonic nanostructures and related applications in light modulators, switches, electronically tuneable spectral filters, and diffraction gratings.

In this paper, we show that the optical transmission through plasmonic crystals fabricated in a gold film can be controlled by electrical manipulation of the refractive index of an LC layer seen by surface plasmon polaritons. We will show that controlled variations of the director orientation of the liquid crystal in the layer adjacent to the nanostructured metallic film affects plasmonic Bloch modes depending on both the state of polarization and the penetration depth of the Bloch mode's electric field in the liquid crystal layer.

The plasmonic crystals of various geometries were created in gold films of different thickness using focused ion beam milling. To fabricate the gold thin films, magnetron sputtering was first used to deposit an adhesion layer of tantalum pentoxide $\left(\mathrm{Ta}_{2} \mathrm{O}_{5}\right)$ on glass $\left(\mathrm{SiO}_{2}\right)$ substrates followed by gold of the desired thickness. Plastic spacers approximately $60 \mu \mathrm{m}$ thick were used to create the liquid crystal cell between the gold film and the indium tin oxide (ITO) glass used as a top electrode (Figure 1). The E7 liquid crystal (Merck) was used without further processing. Ordinary and extraordinary refractive indices, perpendicular and along the LC orientation, are $n_{0}=1.525$ and $n_{\mathrm{e}}=1.734$, respectively.

For optical characterization, spectroscopy was carried out using plane-polarized, collimated white light provided by a tungsten halogen lamp. Light transmitted by the sample was collected using a high-magnification objective lens and divided by a nonpolarizing beamsplitter. This provided light to a charge-coupled device (CCD) video camera enabling surface inspection and focal maintenance while the reflected signal light was coupled into an optical fiber with a $100 \mu \mathrm{m}$ core using a low-magnification objective lens facilitating the acquisition of spatially localized transmission spectra (approximately $10 \mu \mathrm{m}$ in diameter), which were recorded using a spectrometer coupled to a liquid nitrogen cooled CCD array (Figure 1). To obtain dispersion plots, transmission spectra, normalized to the sensitivity of the system and light source, were measured at a series of incident angles (every $2^{\circ}$ ). Polynomial interpolation was performed at each frequency in the acquired spectra relating the transmitted intensity to the vector $k_{x}$. The dispersion was then generated by recalculating this polynomial within the measured range of $k_{x}$ for each frequency.

Figure 2 shows the scanning electron microcscopy (SEM) images of three different SPP crystals fabricated, using focused ion beam (FIB) milling, on an optically thick gold film (thickness $200 \mathrm{~nm}$ ). These structures comprise both one (1D) and two-dimensional (2D) arrays of subwavelength apertures having the same period $(550 \mathrm{~nm})$. In the 1D case, the array consists of lines having a width of approximately $100 \mathrm{~nm}$. For the 2D structures, square arrays have been created using either a circular hole (diameter $180 \mathrm{~nm}$ ) or a rectangle (200 $\mathrm{nm}$ length, $100 \mathrm{~nm}$ width) as the lattice basis. The dispersions plots $\hbar \omega=f\left(k_{\|}\right)$of the zero-order optical transmission of these nanostructures are shown in Figure 2 when the static electric field controlling the LC's orientation in the cell takes the values of $0 \mathrm{kV} / \mathrm{cm}$ (OFF state) or 12.5 $\mathrm{kV} / \mathrm{cm}$ (ON state). In all measurements, the polarization of the incident light was in the plane of incidence and perpendicular to the lines and the long axis of the rectangles. The voltage-induced changes of the transmission are highlighted in the differential dispersion plots in Figure $2 \mathrm{~d}, \mathrm{~h}, \mathrm{l}$ that represent the differential ratio $\left(\mathrm{T}_{\mathrm{ON}}-\mathrm{T}_{\mathrm{OFF}}\right) / \mathrm{T}_{\mathrm{OFF}}$ between the transmission values when the static electric field is $\mathrm{ON}$ and OFF. It should be noted that the cross-sections of the dispersion plots at different values of the wave vector represent the transmission spectra of the nanostructure at the angle of incidence corresponding to a given $k_{\|}$. When the electric field is applied to the structure both a modification of the magnitude of transmittance at the resonant wavelengths and a spectral shift of the resonances are observed. The changes in the transmission are most important close to the band-edges of the SPP crystals as should be expected. Illumination of the sample from the opposite side gives similar results indicating that pure polarization effects, which are responsible for the electric switching in the transmission of plasmonic nanorod arrays, ${ }^{16}$ are in this case negligible compared to the induced refractive index change, which results in the modification of the SPP dispersion in the plasmonic crystal.

Neglecting the appearance of band-gaps, the dispersion of the optical transmission of SPP crystals can be understood by considering, separately, the effect of a periodic structure and the dispersion of surface plasmons polaritons supported by a smooth interface between a dielectric material of refractive index $\sqrt{\epsilon_{\mathrm{d}}}$ and a semi-infinite metal with the dielectric constant $\epsilon_{\mathrm{Au}}$. This model gives the dispersion bands 

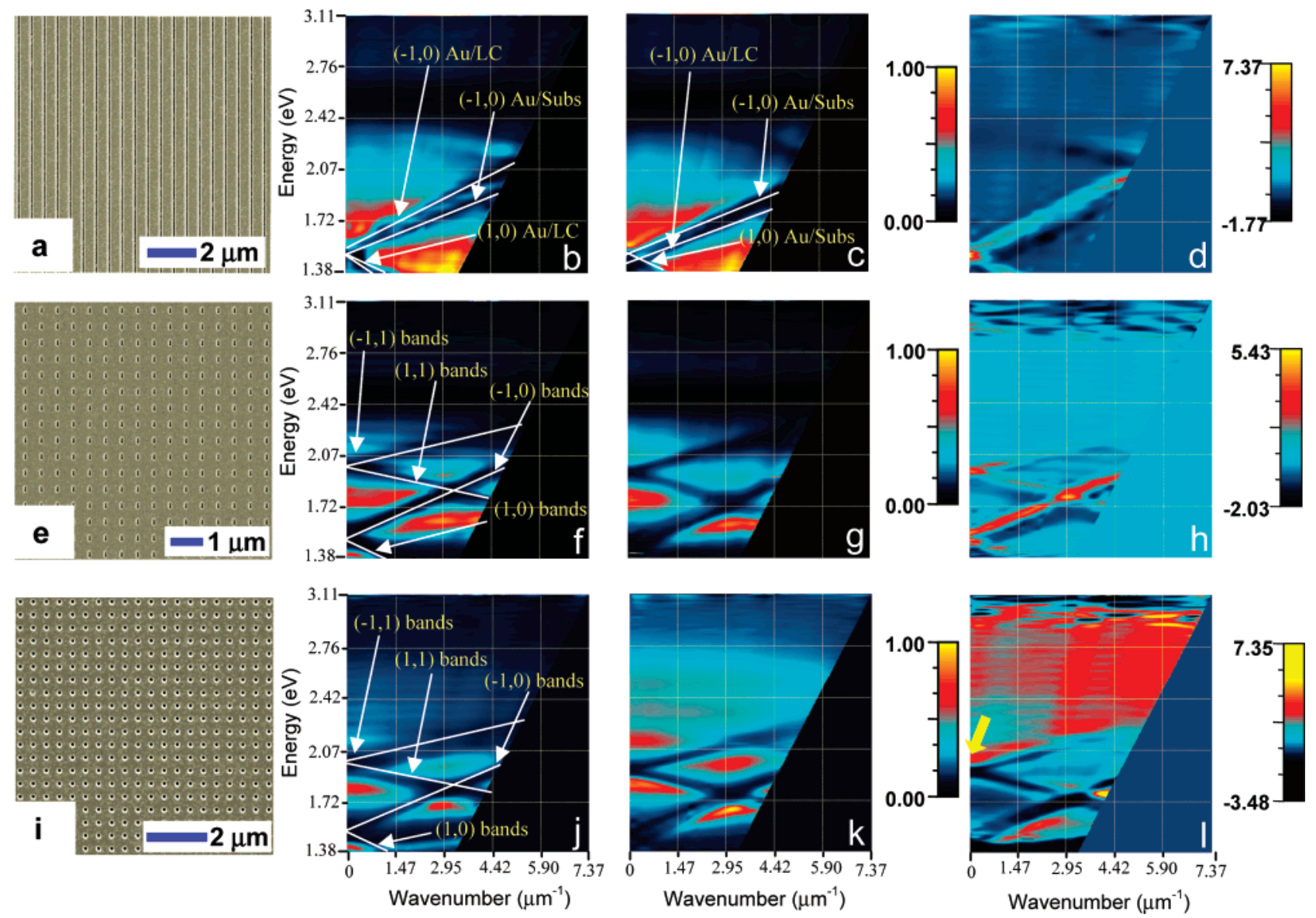

Figure 2. SEM images and optical transmission dispersion plots of the nanostructures in the $200 \mathrm{~nm}$ thick Au film. SEM images for a periodic array of (a) lines (60 nm width, $550 \mathrm{~nm}$ period), (e) rectangles $(200 \mathrm{~nm} \times 50 \mathrm{~nm}, 550 \mathrm{~nm}$ period) and (i) holes (150 nm diameter, $550 \mathrm{~nm}$ period). Optical transmission dispersion plots for the structures in liquid crystal without and with an applied electric field (12.55 $\mathrm{kV} / \mathrm{cm}$ ) are shown in panels $\mathrm{b}$ and $\mathrm{c}$ for the lines, panels $\mathrm{f}$ and $\mathrm{g}$ for the rectangles, and panels $\mathrm{j}$ and $\mathrm{k}$ for the holes, respectively. (d,h,l) These panels show the change in transmission dispersion associated with the application of the applied field for the lines, rectangles, and holes, respectively. The yellow arrow in panel 1 indicates where the dynamic response of the transmission was studied. The white lines are the dispersion calculated with eq 1 and Au permittivity from ref 22. It should be noted that in contrast to the slit array, the SPP bands on the opposite interface in the case of circular and rectangular basis overlap and cannot be distinguished in the plots. Color bar intensities are normalized to 1 for each structure.

close to the minima of the transmission spectrum, because the dispersion of SPPs on a smooth surface always falls between the allowed Bloch modes of the SPP crystal. ${ }^{1}$ For each band, the dispersion can be expressed in this approximation as

$$
\begin{array}{r}
\vec{k}_{\mathrm{SPP}-\mathrm{B}}=\vec{k}_{\mathrm{SPP}}+\vec{k}_{\mathrm{Bragg}}=\frac{2 \pi}{\lambda_{0}} \sqrt{\frac{\epsilon_{\mathrm{Au}}(\omega) \epsilon_{\mathrm{d}}}{\epsilon_{\mathrm{Au}}(\omega)+\epsilon_{\mathrm{d}}}} \vec{u}+l \frac{2 \pi}{d} \vec{i}+ \\
m \frac{2 \pi}{d} \vec{j}
\end{array}
$$

where $k_{\mathrm{SPP}}$ and $k_{\mathrm{SPP}-\mathrm{B}}$ are the SPP wave vectors before and after Bragg scattering in the periodic lattice, $k_{\text {Bragg }}$ is the momentum contributed during the scattering by the crystal, $\lambda_{0}$ is the vacuum wavelength, $\epsilon_{\mathrm{Au}}(\omega)$ and $\epsilon_{\mathrm{d}}$ are the permittivities of $\mathrm{Au}$ and the adjacent dielectric respectively, $d$ is the period of the crystal with the square lattice, $(l, m)$ are integers accounting for Bragg reflected SPPs, $\vec{u}$ is the unit vector describing the SPP propagation direction before Bragg scattering, and $\vec{\imath}$ and $\vec{j}$ are the unit reciprocal lattice vectors of the periodic structure. The different SPP bands are identified in Figure 2 by the values of $(l, m)$ corresponding to the direction in reciprocal space responsible for their scattering and the supporting interface. The dispersion of the 2D crystals (Figure 2e-1) exhibit additional bands compared to the $1 \mathrm{D}$ case (Figure $2 \mathrm{a}-\mathrm{d}$ ) due to extra Bragg scattering channels along the $( \pm 1, \pm 1)$ directions not present in $1 \mathrm{D}$ crystals.

In addition to the effect of Bragg scattering on the crystal lattice described above, the dispersion also depends on the scattering cross-section of the crystal basis (a circular hole, a square hole, or a slit) as well as on the spatial variations of the refractive index of the adjacent medium. ${ }^{17}$ The role of each of these effects will be exemplified when analyzing the response of the crystal to the applied static electric field. However, the general behavior of the optical properties of all SPP crystals studied to a reorganization of the adjacent LC layer is very similar. Therefore, we will focus the discussion on the case of the 1D crystal (Figure 2a) and then generalize the observed behavior to the $2 \mathrm{D}$ case. 
As seen from Figure $2 b$, two SPP Bloch modes that intersect the $k_{\|}=0$ ordinate at around 1.53 and $1.56 \mathrm{eV}$ may be identified. These modes correspond to the $( \pm 1,0)$ SPP bands for the substrate and LC interfaces, respectively [this consideration based on eq 1 holds away (a few tens of $\mu \mathrm{m}^{-1}$ ) from the center of the first Brillouin zone where different bands interfere, inducing the appearance of band gaps]. The respective position of these bands is related to the effective refractive index of these modes at each interface, where the substrate interface presents a higher effective index than the LC interface. ${ }^{18}$ The effective indices of these lowfrequency modes can be estimated using eq 1 substituting $n_{\mathrm{eff}}{ }^{2}=\epsilon_{\mathrm{d}}$ as a parameter. The values for the Au/substrate and $\mathrm{Au} / \mathrm{LC}$ interfaces are then found to be $n_{\text {eff-glass }}=1.42$ and $n_{\text {eff-LC-no }}=1.39$, respectively.

On application of the static electric field, the upper band corresponding to the Au/LC SPP band shifts to lower energy, sweeping across the substrate mode and crossing the center of the first Brillouin zone at around $1.4 \mathrm{eV}$. The corresponding effective index for this mode is in this case approximately $n_{\text {eff-LC-ne }}=1.55$ and corresponds to the LC being preferentially aligned with the static field, that is, perpendicular to the interface. This corresponds to a $0.16(1.55-1.39)$ variation in the effective refractive index of the LC layer while the maximum refractive index variation corresponding to a $90^{\circ}$ reorientation of the LC in the cell is $0.21\left(n_{\mathrm{e}}-n_{\mathrm{o}}\right.$ $=1.734-1.525)$. Considering that the LC molecules are predominantly aligned with the applied field when the voltage is ON and principally aligned with the electrodes interfaces when the voltage is OFF, and taking into account that the lower-frequency SPP Bloch mode on the Au/LC interface has its electric field predominantly normal to the interface, this SPP mode appears to be mostly sensitive to the refractive index variation perpendicular to the metal interface. Additionally, the measurements in Figures $2 b, c$ reflect the spatial sensitivity of the SPP Bloch modes. This is evidenced in the differential dispersion plotted in Figure $2 \mathrm{~d}$. Indeed, this plot shows that the lower SPP band (on the $\mathrm{Au} / \mathrm{LC}$ interface) is most sensitive to changes of the refractive index in the cell rather than in close proximity to the interface. The lower lying bands at the $\mathrm{Au} / \mathrm{LC}$ interface, that is, the $( \pm 1,0)$ bands, have a stronger penetration depth in the LC region than the higher lying bands, which are more confined to the $\mathrm{Au} / \mathrm{LC}$ interface. The spectral position of the latter would then be more sensitive to changes in refractive index occurring in close proximity to the $\mathrm{Au} / \mathrm{LC}$ interface while the former are more sensitive to changes in the bulk, a few hundred nanometers into the LC medium. This behavior is understandable because it is known that the penetration depth of SPPs into the dielectric medium from the metal surface increases with decreasing frequency ${ }^{19}$ leading to the observed higher sensitivity of the lowfrequency modes exhibited by the crystals in Figure 2.

Replacing the slits with holes of rectangular cross-section opens additional scattering channels, in particular along the $( \pm 1, \pm 1$ ) directions (Figure 2f,g). Compared to the dispersion of the 1D crystal, the dispersion of the array of rectangles also reflects the scattering cross-section of the holes. This is evidenced in the bare crystal dispersion (Supporting Information, Figure S1) and in the differential dispersion of Figure $2 \mathrm{~h}$ showing that the $\mathrm{LC}$ reorganization affects not only the lower frequency bands (as for the slits) but also leads to additional dispersion modification at normal incidence at around $1.75 \mathrm{eV}$. This latter feature is related to the changing SPP-scattering properties of the basis subsequent to the LC reorganization in the hole when the static electric field is varied. However, the fact that both the dispersion, as evaluated from eq 1 , as well as the SPP-scattering properties of the holes are sensitive to the LC reorganization in a correlated manner makes it difficult to determine how the SPP-scattering properties of the holes are changing as a function of applied field. Additionally, LC geometries in confined structures can be extremely complicated requiring significant computational power to address them satisfactorily. ${ }^{20,21}$ The contribution of the scattering cross-section of the basis to the differential optical response of the plasmonic crystal is further accentuated for the array of holes with circular cross-section. In this case, the index of refraction change due to LC reorganization leads also to the modification of a localized plasmon mode in the hole influencing the optical properties of the array at the frequency of about $1.84 \mathrm{eV}$ at normal incidence (Figure $2 \mathrm{j}-1$ ). Returning briefly to the spatial sensitivity of the low-frequency SPP bands to changes of the refractive index occurring in the bulk of the $\mathrm{LC}$ region, it is worth noting that the $(1,1)$ band in Figure $2 \mathrm{f}-\mathrm{h}$ is not as sensitive to the LC reorganization as the $( \pm 1,0)$ bands. Although, these bands span the same spectral range in the dispersion, the field associated to the $(1,1)$ band is more confined to the $\mathrm{Au} / \mathrm{LC}$ interface than the $( \pm 1,0)$ band, which is in agreement with our discussion above.

To investigate the dynamic response of the liquid crystal cell with a view on potential applications, the transmission of the array of circular holes (200 nm thick film) was monitored as the cell was subjected to a time-modulated electric potential. The energy and momentum of the incident light were chosen such as to probe the mode at $k_{\|}=0 \mu \mathrm{m}^{-1}$, $\hbar \omega=1.85 \mathrm{eV}$ corresponding to a vacuum wavelength of $670 \mathrm{~nm}$, where a large variation of transmitted light intensity was observed on application of the electric field (indicated by the yellow arrow in Figure 2d). The change in optical transmission with increasing applied electric field is illustrated by the color plot shown in Figure 3a: the conformational change of the liquid crystal layer can be seen to take place between 0.4 and $5 \mathrm{kV} / \mathrm{cm}$, and within this region the transmission is unstable. Both the spectral shift and change in amplitude of the transmission peaks are also clearly visible with applied field (Figure 3b). The observed dynamic response of the transmission under the temporal modulation of the applied electric field at frequencies of 2.5 and 100 $\mathrm{Hz}$ demonstrate the ability of the structure to switch and modulate light at a frequency corresponding to that of today's modern LC displays with less than a $10 \%$ reduction in amplitude.

The coupling of SPP modes on the opposite interfaces of a thin metal film is an additional factor contributing to the SPP Bloch mode dispersion and thus influences the switching 

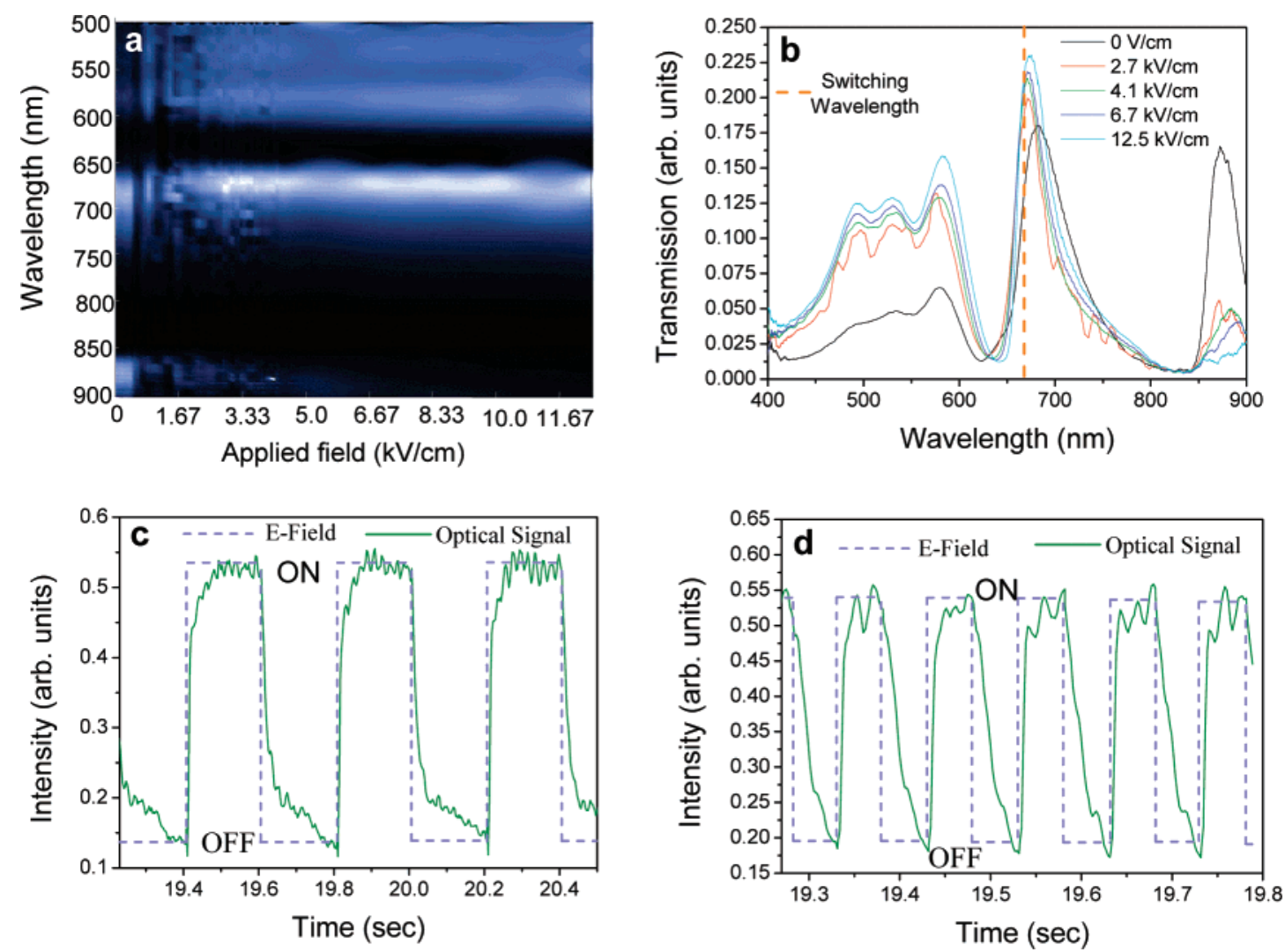

Figure 3. Electric field dependence of the optical transmission for the $2 \mathrm{D}$ array of nanoholes shown in Figure 2. (a) The change in transmission (color plot) for increasing applied electric field. (b) Transmission spectra for several values of applied electric field. The wavelength used to probe the switching dynamics is shown by a dashed orange line. (c,d) show the transmitted light intensity at a wavelength of $670 \mathrm{~nm}$ through the periodic array of holes as the potential across the liquid crystal cell is modulated at $2.5 \mathrm{and} 100 \mathrm{~Hz}$ respectively. All measurements were performed at normal incidence.
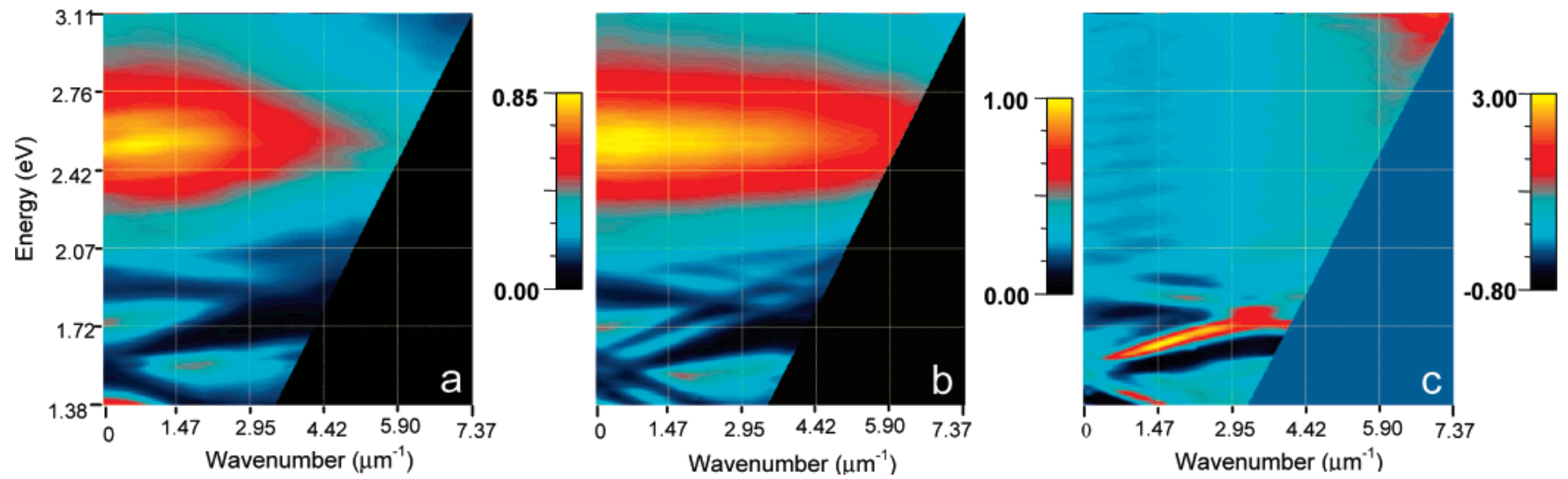

Figure 4. Experimentally measured optical transmission dispersion for a periodic array of nanoholes (diameter $150 \mathrm{~nm}$, period $600 \mathrm{~nm}$ ) in a $50 \mathrm{~nm}$ gold film in LC (a) with no applied field, (b) with electric field applied, and (c) showing the change in dispersion of the structure on application of the field.

behavior. To investigate this influence, structures similar to those previously created were fabricated in a $50 \mathrm{~nm}$ thick gold film. Similar effects of the LC switching have been observed (Figure 4). The double structure of the bands due to the coupling of the modes can be observed in the differential transmission dispersion. In contrast to the thick $\mathrm{Au}$ films, the high-frequency SPP bands exhibit stronger sensitivity to the applied electric field because the spatial field extension in the LC layer is stronger for SPP modes supported by thinner films. ${ }^{1}$
In conclusion, we have demonstrated the possibility to control the dispersion of surface plasmon waves on surface plasmon polaritonic crystals and related optical transmission electronically using liquid crystal molecules. The observed modification of the dispersion is directly related to the changes in effective refractive index of the superstrate as seen by the SPP Bloch waves, caused by a reorientation of the liquid crystal director on application of an electric field. The feasibility of employing such a structure in a dynamic device has been confirmed by light-switching experiments. 
Despite the limitations imposed by the response time of the liquid crystal molecules, the ability to tune or modulate the optical response of the structure together with the flexibility to tailor the optical response of the array (by variation of the array periodicity and geometry of the lattice basis) suggests potential applications in display technologies. Additionally, the possibility exists to design plasmonic structures to perform complex optical signal processing and pulse shaping as well as design plasmonic metamaterials with electrically tuneable negative refraction or "perfect lensing" properties.

Acknowledgment. This work was supported in part by EPSRC (UK) and EC FP6 STREP PLASMOCOM. The authors are grateful to W. Hendren for help with the LC cell fabrication and D. O'Connor for help with numerical modeling.

Supporting Information Available: Included are dispersion plots for all the plasmonic crystals used in this work with air as the superstrate and in addition the numerically calculated dispersions plots for an array of lines (period 550 $\mathrm{nm}$, width $100 \mathrm{~nm}$ ) with air and liquid crystal (ON and OFF) as the superstrate.

\section{References}

(1) Zayats, A. V.; Smolyaninov, I. I.; Maradudin, A. A. Phys. Rep. 2005, 408, 131.

(2) Ozbay, E. Science 2006, 311, 189.

(3) Ebbesen, T. W.; Lezec, H. J.; Ghaemi, H. F.; Thio, T.; Wolff, P. A. Nature 1998, 391, 667.

(4) Barnes, W. L.; Dereux, A.; Ebbesen, T. Nature 2003, 424, 824.

(5) Smolyaninov, I. I.; Hung, Y.-J.; Davis, C. C. Science 2007, 315, 1699.
(6) Lezec, H. J.; Dionne, J. A.; Atwater, H. A. Science 2007, 316, 430

(7) Cai, W.; Chettiar, U. K.; Kildishev, A. V.; Shalaev, V. M. Nat. Photonics 2007, 1, 224.

(8) Wegener, M.; Dolling, G.; Linden, S. Nature Mat. 2007, 6, 475.

(9) Dickson, W.; Wurtz, G. A.; Evans, P.; O'Connor, D.; Atkinson, R.; Pollard, R.; Zayats, A. V. Phys. Rev. B. 2007, 76, 115411.

(10) Wurtz, G. A.; Pollard, R.; Zayats, A. V. Phys. Rev. Lett. 2006, 97, 057402.

(11) Pacifici, D.; Lezec, H. J.; Atwater, H. A. Nat. Photonics 2007, 1, 402.

(12) Nikolajsen, T.; Leosson, K.; Bozhevolnyi, S. I. Appl. Phys. Lett. 2004, 85,5833

(13) Wang, Y. Appl. Phys. Lett. 1995, 67, 2759.

(14) (a) Kossayev, P. A.; Yin, A.; Cloutier, S. G.; Cardimona, D. A.; Huang, D.; Alsing, P. M.; Xu, J. M. Nano. Lett. 2005, 5, 1978. (b) Park, S. Y.; Stroud, D. Phys. Rev. Lett. 2005, 94, 217401.

(15) Chu, K. C.; Chao, C. Y.; Chen, Y. F.; Wu, Y. C.; Chen, C. C. Appl. Phys. Lett. 2006, 89, 103107.

(16) Evans, P. R.; Wurtz, G. A.; Hendren, W. R.; Atkinson, R.; Dickson, W.; Zayats, A. V.; Pollard, R. J. Appl. Phys. Lett. 2007, 91, 043101.

(17) Numerical simulations using finite element method reproduce the measured transmission dispersion and its dependence on structural parameters (see Supporting Information, S2).

(18) Considering the refractive index of glass $(n=1.5)$ and the expected orientation of the LC $\left(n_{\mathrm{e}}=1.735\right.$ along the metal/LC interface and $n_{0}=1.525$ perpendicular to it), the $\mathrm{Au} / \mathrm{LC}$ band should lie at a lower frequency than the Au/glass band. However, the Au film is sputtered on a $10 \mathrm{~nm}$ thick $\mathrm{Ta}_{2} \mathrm{O}_{5}$ base layer with a refractive index of 2 , sufficient to displace the $\mathrm{Au} /$ substrate band below the $\mathrm{Au} / \mathrm{LC}$ band. This is further confirmed by numerical simulations (S2) and when analyzing the response of these bands to an applied field (Figure $2 \mathrm{c}$ ), as only the $\mathrm{Au} / \mathrm{LC}$ band is sensitive to the reorientation of the $\mathrm{LC}$ in the cell.

(19) Barnes, W. L. J. Opt. A: Pure Appl. Opt. 2006, 8, S87.

(20) Steinhart, M.; Zimmermann, S.; Göring, P.; Schaper, A. K.; Gösele, U.; Weder, C.; Wendorff, J. H. Nano Lett. 2005, 5, 429.

(21) Priezjev, N.; Pelcovits, R. A. Phys. Rev. E: 2000, 62, 6734.

(22) Johnson, P. B.; Christy, R. W. Phys. Rev. B 1972, 6, 4370.

NL072613G 